\title{
Verbale Tretminen und Streubomben
}

Bernhard Gurtner

\section{Sprech-Stunden im wahren Wortsinn sind selbst nach Einführung wirksamer Psychopharmaka das wichtigste therapeutische Instrument in der Psychiatrie geblieben.}

Korrespondenz:

Dr. med. Bernhard Gurtner Eggstrasse 76 CH-8620 Wetzikon

gurtner.bernhard[at]bluewin.ch
«Ich kann mit Worten heilen» stand vor 2500 Jahren am Marktplatz von Korinth auf dem Praxisschild des Sophisten Antiphon, der nach heutigen Begriffen als Psychotherapeut tätig war. Durch Fragen half er den Patienten, die Ursachen ihres Kummers zu finden, er deutete ihre Träume und gab ihnen Anregungen für eine gesunde Lebensführung [1]

Jahrhundertelang blieb das ärztliche Wort eines der wirksamsten Heilmittel, weil das hochentwickelte technische Arsenal noch nicht zur Verfügung stand. Sprech-Stunden im wahren Wortsinn sind selbst nach Einführung wirksamer Psychopharmaka das wichtigste therapeutische Instrument in der Psychiatrie geblieben. Doch auch in jedem anderen medizinischen Fachbereich schätzen es die Hilfesuchenden, wenn zuerst gesprochen und dann erst gestochen wird.

Ärztliche Worte können aber ungewollt toxisch wirken und den Patienten oder ihren Angehörigen Leid antun [2]. In zwei eigenen Fällen hatten sie sogar tödliche Folgen: Ein oft jähzorniger Familientyrann - hospitalisiert wegen eines schweren Lungenemphysems - wurde auf der Chefarztvisite scharf gerügt, weil er sich gegenüber den Krankenschwestern wiederholt unflätig benommen hatte. Darauf zwängte er sich durch ein schmales Fenster des Vierer-Zimmers und sprang vom Hochhaus in die Tiefe. Nach ehrlicher Information über die Umstände des bestürzenden Vorfalls erhielten wir einen von der Witwe und fünf erwachsenen Kindern unterzeichneten Brief, der unser Spital von jeder Schuld entlastete. Die Staatsanwaltschaft verzichtete auf ein juristisches Nachspiel. Ein anderes Mal hat die etwas hastige Bekanntgabe der Krebsdiagnose zum Suizid einer Patientin im Dorfweiher beigetragen. Damals fand ein Pfarrer die rechten Worte.

Mediziner haben eine martialische Sprache, weil ihre frühesten Vorfahren als Militärärzte tätig waren. Noch heute gibt es Pikettzimmer, obwohl die Diensttuenden bei Alarm nicht zu den Waffen greifen. Doch die Radiotherapeuten bringen Kobalt-Kanonen in Stellung, Krebszellen werden mit Strahlen be-

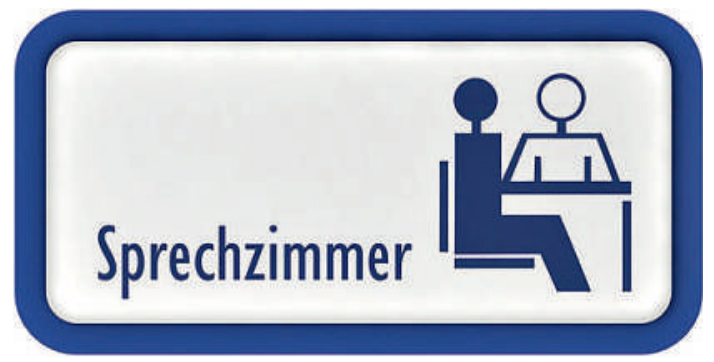

Wird hier zuerst gesprochen und dann erst gestochen?

schossen oder mit Cytostatikastössen ausgerottet. Radikalkuren und invasive Methoden gehören ebenso zum unsanften Vokabular der aggressive Care [3]. Im Zeughaus der polemischen Metaphern lagern aber auch verbale Tretminen und Streubomben.

Verbale Tretminen sind beiläufige Bemerkungen, die im Umgang mit Patienten oder deren Angehörigen unbedacht angebracht werden und das Vertrauen erschüttern oder ganz zerstören. Das kann im Gespräch unter vier Augen geschehen, viel häufiger aber sind es gruppenweise Arztvisiten oder Übergaberapporte im Beisein der Betroffenen, die Missverständnisse und zusätzliche Ängste auslösen. Oft muss das Pflegepersonal verstörte Kranke trösten, beruhigen und aufklären, wenn der weisse, aber nicht immer weise Stosstrupp ins nächste Zimmer abmarschiert ist.

Eine nicht zu unterschätzende Sprengkraft haben die einsilbigen Wörter noch und schon: «Können Sie noch schlucken? Brauchen Sie für die Körperpflege noch keine Hilfe? Haben Sie schon blutigen Auswurf? Haben Sie schon Gefühlsstörungen bemerkt?»

Da ahnt doch jede(r) schon, was ihm noch bevorsteht!

Als verbale Tretminen wirken lateinische, griechische und englische Fachausdrücke, wenn ihre Bedeutung nicht erklärt wird. Unter Tumor verstehen die meisten Nichtmediziner einen Krebs, obwohl es ja auch gutartige Formen gibt. Positive Befunde verschleiern negative Nachrichten. Die dem behandelnden Team wohlvertrauten Abkürzungen sind es keineswegs für die Angesprochenen. Einst verängstigten wir die Patienten mit der Verordnung des Holzknechts oder eines Ivaupehs, heute wissen sie vom Fernsehen Bescheid über Stents und MRI, wagen aber nicht nachzufragen, wenn von erhöhten Transami-Nasen und einem Ehär-zeh-peh die Rede ist. 


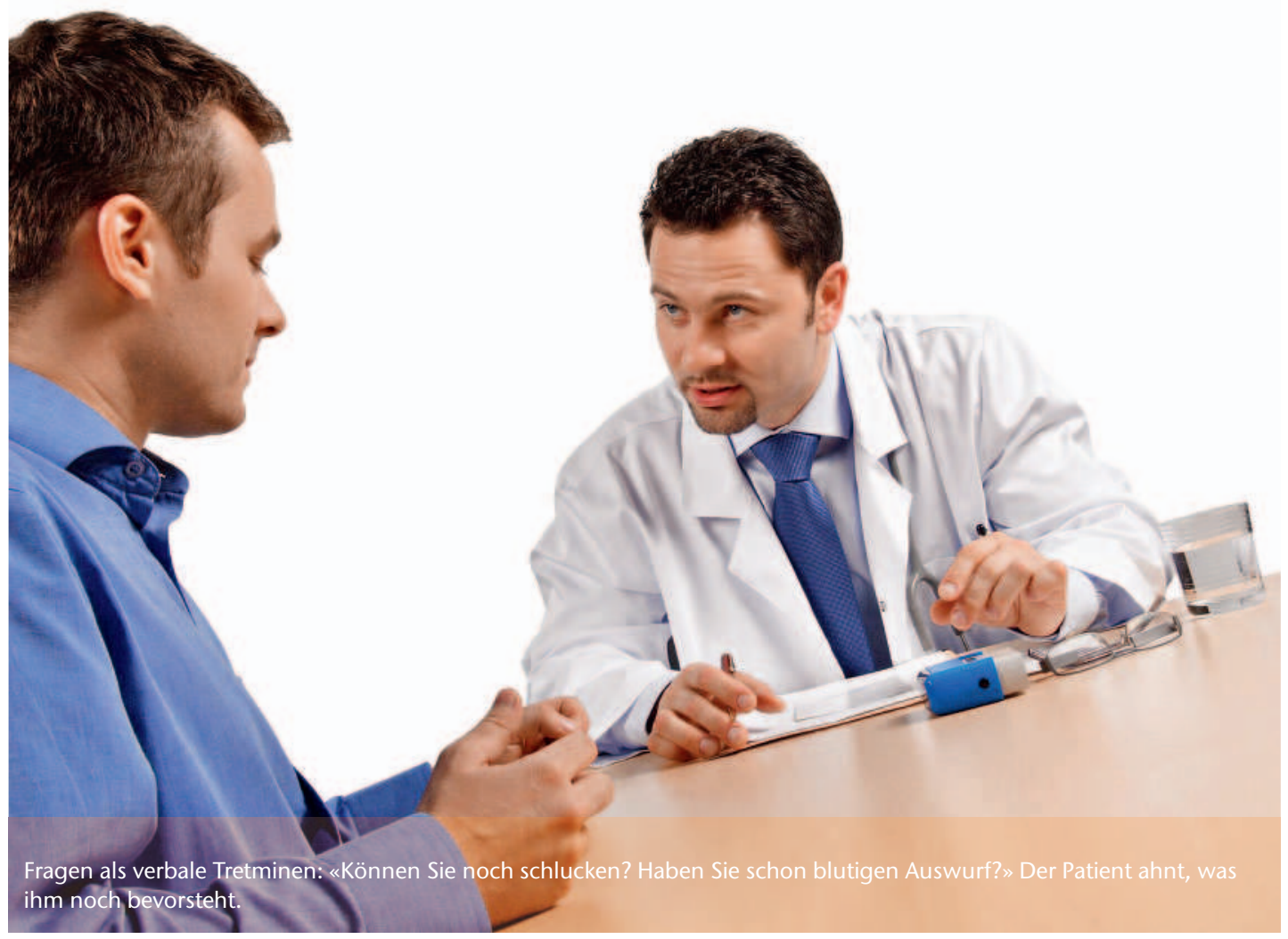

Besondere Rücksicht müsste man auf fremdsprachige Patienten nehmen, bei denen Missverständnisse fast unvermeidlich sind. Im klinischen Alltag stösst man da aber rasch an die Grenzen des Möglichen und darf sich nicht unbedingt auf Dolmetscherdienste der Angehörigen verlassen, die ihre eigenen Interpretationen und Ängste hineinmischen.

\section{Oft muss das Pflegepersonal verstörte Kranke beruhigen und aufklären, wenn der weisse, aber nicht immer weise Stosstrupp ins nächste Zimmer abmarschiert ist.}

Bedside teaching ist eine feine Sache, birgt aber die Gefahr in sich, dass die Kranken zu unbeteiligten Lehrobjekten werden, die nicht verstehen, was über sie gesprochen wird. Sie erschrecken, wenn der Kaderarzt mit einer ironischen Bemerkung das Gelächter seiner Gefolgschaft provoziert, und wundern sich, dass ihre liebe Assistenzärztin die Fragen des Chefarztes nicht beantworten konnte. Sie bedauern und trösten sie oder entziehen ihr das AAA-Vertrauen. Um niemanden blosszustellen, befragen deshalb vorsichtige Ausbildner nur die Unterassistenten, die sich als Studenten noch im Gnadenstand des erlaubten Nichtwissens befinden.

Wenn aufgeregte Teammitglieder hereinstürmen, um mitten in ärztliche Besprechungen zu mel- den, was wieder einmal schiefgelaufen ist, vergessen oder verwechselt wurde, so übertragen sie mit verbalen Streubomben ihren Ärger auf viele Unbeteiligte. Restaurantgäste möchten beim gediegenen Essen auch nicht durch Küchengeräusche, laute Zurufe des Personals oder dessen private Diskussionen gestört werden. Etwas gar sorglos wird da und dort in allgemein zugänglichen Cafeterias und Aufzügen von Spitalangestellten über interne Vorkommnisse diskutiert und gelästert. Sie liefern Munition für Gerüchte und öffentliche Kritik, die sich auf der Heimfahrt in der S-Bahn weiterverbreiten.

Vermint mit Spätzündern schienen oft interdisziplinäre Diskussionen auf Intensivstationen, wo zu Unrecht davon ausgegangen wurde, dass der intubierte Patient oder die im Vorraum wartenden Angehörigen nichts mitbekamen. Die Animositäten zwischen Anästhesisten, Chirurgen und Internisten liessen vermuten, dass nicht immer die Suche nach der besten, erfahrungsgestützten Lösung das Hauptanliegen war, - doch das sind nicht signifikante Beobachtungen aus dem letzten Jahrhundert.

\section{Literatur}

1 Furley William D. Antiphon der Athener: Ein Sophist als Psychotherapeut? www.rhm.uni-koeln.de/135/ Furley.pdf

2 Von Rechenberg HK. Schädigungen durch das ärztliche Wort. Documenta Geigy. 1969.

3 Lütolf UM. Kanonen, Krieg und Krankheit. Schweiz Ärztezeitung. 2000;81(46):2610. 